\title{
Neonatal Isolation Stress Inhibits Pre-Weaning Weight Gain and Mild-Stressor Induced Locomotor Activity in Early Adolescent Male and Female Rats
}

\author{
Peter Villavecchia, Mindy J. D. Miserendino \\ Department of Psychology, Sacred Hear University, Fairfield, USA \\ Email: miserendinom@sacredheart.edu
}

Received 17 June 2015; accepted 14 July 2015; published 17 July 2015

Copyright (C) 2015 by authors and Scientific Research Publishing Inc.

This work is licensed under the Creative Commons Attribution International License (CC BY). http://creativecommons.org/licenses/by/4.0/

(c) (i) Open Access

\section{Abstract}

The present study looked at the effects of neonatal isolation, an early life stress experience, in male and female early adolescent rats, an age which is underrepresented in the early stress literature. Four stress-sensitive indices were assessed: weight gain during the pre-weaning period, open field activity, and locomotor activity in response to two mild stressors: exposure to a novel environment, and a single IP saline injection. Rats in the neonatal isolation condition were removed from dam and littermates on postnatal days 2-9 in accord with the procedure used by Kehoe et al. (1995); behavioral testing occurred on PN25-PN30 during the early adolescent period. It was found that neonatally isolated pups weighed less than non-isolate controls on each of three measurement days (PN7, PN14, PN21) throughout the pre-weaning period. Further, neonatal isolation experience consistently reduced horizontal locomotor activity measured in the open field, in a novel environment, and following a single mild acute stressor. On some measures, behavior reflected greater impact of NI in males compared with NI females, suggesting that the effects of NI in early adolescent rats may be sexually dimorphic.

\section{Keywords}

Neonatal Isolation, Early Life Stress, Adolescent, Sex Differences, Open Field Test 


\section{Introduction}

Adverse experiences very early in life impact the development of neurochemical, neurophysiological and neuroendocrine systems, resulting in altered function of those systems later in life [1]-[3]. To observe and examine the effects of early life stress on those neurobiological systems and the behaviors they subserve, animal models such as maternal separation and neonatal isolation are used. Maternal separation (MS) paradigms involve removal of the pups from the dam for some duration of time while allowing the pups to remain in a huddle, thus preserving the interpup olfactory, tactile, and auditory stimuli. Neonatal isolation (NI), by contrast, involves removal of the pups from the dam and also from each other in a controlled environment for some duration of time each day over several days. Both models significantly disrupt normal dam-pup interactions throughout much or all of the early postnatal period [4], including the stress hypo-responsive period (SHRP) in the first 2 weeks of life [5]. Stressors experienced during this developmentally sensitive period produce long-term neurobiological changes that last into adulthood [6] [7].

The widely varying methodologies used in MS, and, notably, the presence versus absence of littermates in MS compared with NI, provide very different experiential stressors [8]. Thus MS studies often produce different neurochemical, hormonal and behavioral outcomes compared with one another, and compared with studies using NI. Allowing for procedural variants of MS to affect the magnitude and/or direction of the early stress effects, both early stress models in general exert widespread and enduring neurobiological and behavioral effects. Early life stress effects include, but are not limited to, changes in HPA reactivity and behavioral responses to stress [9]-[15], neurochemical alterations, including some that impact vulnerability to drugs of abuse [15]-[22], and hippocampal modifications that impair or enhance learning and memory [23]-[26].

Given the disparate outcomes resulting from quite diverse procedures subsumed under the rubric of maternal separation [27], the current studies instead examined the effects of the neonatal isolation paradigm, specifically as described and utilized by Kehoe and colleagues [28].

Most of the literature addressing early life stress focuses on neurobiological or behavioral changes in adult rats; other age points, such as pups, adolescents, or aged rats are far less often studied [23]. Given the continual maturation of all aspects of the CNS, including the HPA axis [29], the effects of an early life stressor may be expected to vary as a function of age and maturational stage of assessment when observed in pups, weanlings, older adolescents, or fully adult rats [30]. Some studies on neonatal isolation stress effects in pups and adolescent rats have been done, primarily by Kehoe and colleagues [28] [31]-[34] but far less information exists on early life stressor induced effects in these age groups. When compared with adults, adolescents often show differences in neurobiological and behavioral indices of both basal and stressor-induced anxiety [35]. Thus extrapolations cannot simply be made from findings in adults to predict early life stress effects in adolescent rats. For these reasons, the present studies were designed to extend the current literature on early life stress effects in early adolescent rats by examining the effects of neonatal isolation on behavioral responses to several mild stressors during the early adolescent period in both male and female rats.

\section{Methods}

\subsection{General Methods}

Subjects: Twenty three pregnant dams (Charles River, Sprague Dawley) arrived at G16, were individually housed in standard plastic bedding cages, and were monitored daily to determine date of birth, PN0. On PN2, pups from each of 23 litters litter were weighed, sexed, and counted. Litters were culled to $\mathrm{N}=10 \mathrm{pups} / \mathrm{litter}$, with as near an even distribution of 5 males and 5 females/litter as possible. Odd number litters were assigned to the Neonatal Isolation (NI) condition; even number litters to the Non-Isolation (NO NI) condition, resulting in 12 NI and 11 NO NI litters. Rat pups lived with their dam and siblings in standard plastic bedding cages in a temperature controlled colony room maintained on a 12:12 light/dark cycle (lights on at 8:00 am) until weaning at PN21. At weaning, pups were sorted into new plastic bedding cages with $\mathrm{N}=3$ or 4 rats/cage forming 4 experimental groups: NI males, NI females, NO NI males, and NO NI females. Subsets of each of these 4 groups were randomly assigned to participate in the experiment reported here, and several others.

All animals had food and water available ad lib throughout the experiment, and all procedures were approved by the Institutional Animal Care and Use Committee (IACUC) at Sacred Heart University in compliance with the NIH Guide for the Care and Use of Laboratory Animals. 


\subsection{Apparatus and Procedures}

\subsubsection{Neonatal Isolation (NI)}

The neonatal isolation procedure has been described previously [28]. Briefly, with the day of birth designated PN0, all pups in litters assigned to the NI condition began the 8 day isolation procedure on PN2 and continued daily through PN9. On each isolation day, each pup designated NI was removed from dam and littermates and placed in a small individual opaque plastic container with bedding located in a water bath containing 2 inches of warm water. The temperature of the bath was continually monitored and held at $30^{\circ} \mathrm{C}$ degrees. After one hour, pups were returned to the dam in the home cage. NO NI condition pups remained undisturbed in the home cage with the dam and litter throughout the pre-weaning period; NI pups remained undisturbed with dam and littermates after PN9. Both NI and NO NI groups experienced periodic weighing (as below) and cage cleaning until weaning at PN21.

\subsubsection{Weights}

Pups from the total cohort of NI and NO NI animals were designated for several ongoing studies, thus the number of animals weighed varies among assessment days. Animals were weighed after litters were culled on PN2 $(\mathrm{N}=170)$, and weighed again on PN7 $(\mathrm{N}=110)$, PN14 $(\mathrm{N}=168)$ and at weaning on PN21 $(\mathrm{N}=118)$.

\subsubsection{Behavioral Testing}

All behavioral testing occurred during prepubescence/early adolescence as defined [29] between PN26 and PN30, inclusive. Each rat was first tested in the Open Field, followed by one day off, then was tested for locomotor activity in the Novel circular corridor. After another day off, a random subset of the animals used for the Novel circular locomotor test received an intraperitoneal (IP) saline injection $(1 \mathrm{ml} / \mathrm{kg})$ and each was returned immediately after injection to the circular corridor for a second assessment of locomotor activity. After each rat was tested in the open field or circular corridor, the apparatus was cleaned with a 70\% alcohol solution.

\subsubsection{Open Field Test}

The open field has long and often been used to measure anxiety and to assess anxiolytics in rodents [36]-[38]. In the current study the open field was a rectangular enclosure measuring 50" $\times 30$ " and evenly illuminated by ambient light. The floor of the field was marked off in fifteen 10" square boxes arranged in a $3 \times 5$ grid such that 12 boxes comprised the perimeter and 3 boxes were designated the center area of the field. Each rat began a 6 minute trial by being placed individually into the field in the back right corner facing the center. Horizontal locomotor activity was assessed as the number of box entries, with an entry defined as placing any 2 paws completely into a box. Entries for the total, perimeter, and center field boxes were recorded.

Anxiety and stress are inversely related to activity in the open field, such that lower levels of stress are indicated by greater amounts of horizontal and vertical exploratory behavior. Additionally, since rats have a strong tendency to stay near the perimeter of an open area, more time spent or greater distance traveled in the central area of the field is also thought to indicate lower anxiety [39].

\subsubsection{Novel Circular Corridor Locomotor Test}

Since a novel environment serves as a mild stressor and evokes an anxiety response in rats [38] rats were assessed for levels of horizontal locomotor activity in a novel environment, a circular locomotor corridor, designed after Piazza et al. (1989; [40]). The circular corridor was comprised of 2 concentric heavy plastic opaque rings 24 " inches high. The outer and inner rings were respectively 26 " and 18" in diameter, forming a 4 " wide circular corridor between the rings for the rat to traverse. A photocell sensor was located at each of the 4 cardinal directions of the circular locomotor apparatus, and one locomotor count occurred each time one of the sensor beams was interrupted by the rat's movement. Test sessions were controlled and horizontal locomotor activity data were collected on each rat over a single 30 minute trial using Graphic State Notation computer software interfaced with Coulbourn instrumentation.

\subsubsection{Saline Injection Stressor Challenge}

One day after the initial test of locomotor activity in the novel circular locomotor environment, a random subset of rats from each of the four conditions was selected and challenged with an IP injection $(1 \mathrm{ml} / \mathrm{kg})$ of saline, a mild stressor [41]. Immediately after injection each rat was placed in the circular corridor locomotor apparatus 
as before, and horizontal locomotor activity was again recorded for 30 minutes.

\section{Results}

Neonatally isolated (NI) and non-Isolated (NO NI) Males and Females were assessed on four dependent measures. Prior to behavioral testing, animals were weighed on PN2, PN7, PN14, and PN21 to examine effects of NI on body weight during the pre-weaning period. Behavior was measured over 5 days during adolescence (PN26 through PN30) and included total horizontal activity in the open field, locomotor activity in a novel circular corridor, and locomotor activity measured a second time in the same circular corridor immediately following administration of a mild stressor (IP saline injection).

Weight data at each age point, open field activity, and novel circular corridor and post-injection stress locomotor activity were all analyzed using a 2 way ANOVA with Sex (male, female) and Environment (NI, NO NI) as independent factors. Post-hoc analyses were conducted using a Bonferroni test.

\subsection{Weights}

PN2. Prior to culling litters on PN2, 90 animals from odd number litters assigned to be in the NI Environment condition, and 80 animals from even number litters assigned to be in the NO NI Environment condition, were weighed. There was no main effect of the Environment manipulation that would be subsequently administered, that is, there was no baseline weight difference in the to-be NI and NO NI pups. Weights taken on PN2 did reveal a main effect of Sex $(F(1,166)=9.983, p=0.002)$ with male pups weighing more than females (see Figure 1(a)).

PN7. Several litters were not weighed at PN7. For those litters on which data was collected there was a main effect of Environment $(F(1,106)=40.730, p<0.0001)$, indicating that overall, NI animals weighed less than NO NI animals at PN7. Both females and males showed this same effect of Environment; NI females weighed less than NO NI females, and NI males weighed less than NO NI males $(F(1,51)=34.818, p<0.0001 ; \mathrm{F}(1,55)$ $=48.147, p=0.002$, respectively; see Figure 1(b)). No main effect of Sex was seen at PN7.

PN14. At PN14there was a main effect of Environment $(\mathrm{F}(1,164)=3.903, p=0.050)$ showing that NI animals again weighed less than NO NI animals. Additionally, males were heavier than females at PN14 $(\mathrm{F}(1,164)$ $=4.946, p=0.028$; see Figure $1(\mathrm{c})$ ).

PN21. Several litters were not weighed at weaning, PN21. For those litters on which data was collected, overall, animals showed a main effect of Environment with NI animals weighing less than NO NI animals $(\mathrm{F}(1,114)$ $=7.449, p=0.007$. It was also seen that males were heavier than females at PN21, $(\mathrm{F}(1,114)=4.422, p=0.038$; see Figure 1(d)).

\subsection{Behavioral Measures}

\subsubsection{Open Field Activity}

Only a trend $(p=0.057)$ for an effect of Environment on Open Field activity was seen, with NI animals showing less activity than NO NI animals. A 2 way ANOVA was used to further examined a significant Sex X Environment interaction $(\mathrm{F}(1,143)=12.723, p<0.0001)$ to look at NI effects in males and females. There was no effect of Environment on total open field activity in females; however, NI Males engaged in far less total activity in the open field than NO NI males, $(\mathrm{F}(1,67)=15.516, p<0.0001$; see Figure 2). An additional 2 way ANOVA looked at possible sex differences within each Environment condition. Interestingly, it was observed that in the NO NI condition, Males were more active than Females, $(\mathrm{F}(1,67)=6.277, p=0.015)$, but the reverse was true in the NI condition with Females having more activity in the open field than Males, $(\mathrm{F}(1,76)=6.391, p=0.014$; see Figure 2).

\subsubsection{Locomotor Activity in Response to a Novel Environment, the Circular Corridor}

Total locomotor activity during a 30 minute test session in the novel circular locomotor apparatus was examined. A main effect of Environment indicated that in response to the mild stress of exposure to a novel environment, $\mathrm{NI}$ animals engaged in less total horizontal locomotor activity than NO NI animals $(\mathrm{F}(1,142)=6.360, p=0.013$; see Figure 3).

\subsubsection{Locomotor Activity Following a Mild Stressor}

A subset $(\mathrm{N}=48)$ of the animals used to test activity in the novel circular corridor, above, was administered a 
NI and NO NI animals are equal in weight at PN2

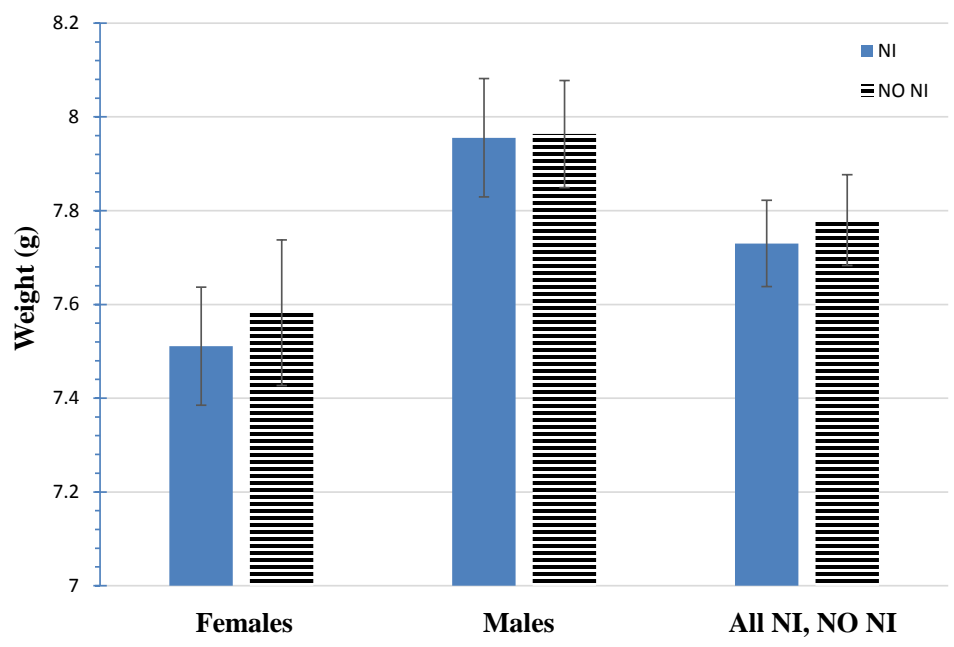

(a)

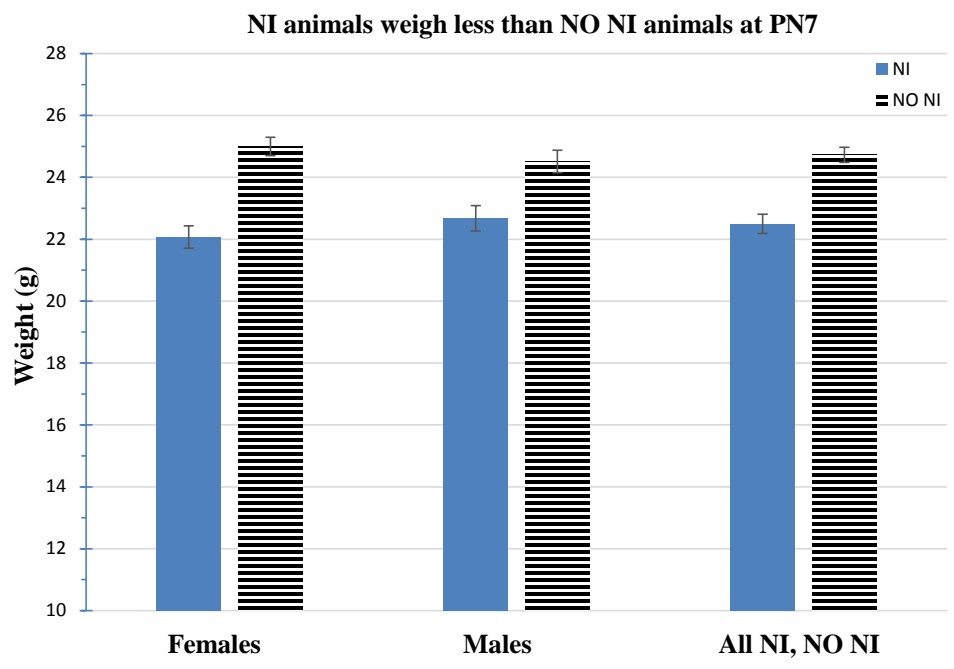

(b)

NI animals weigh less than NO NI animals at PN14

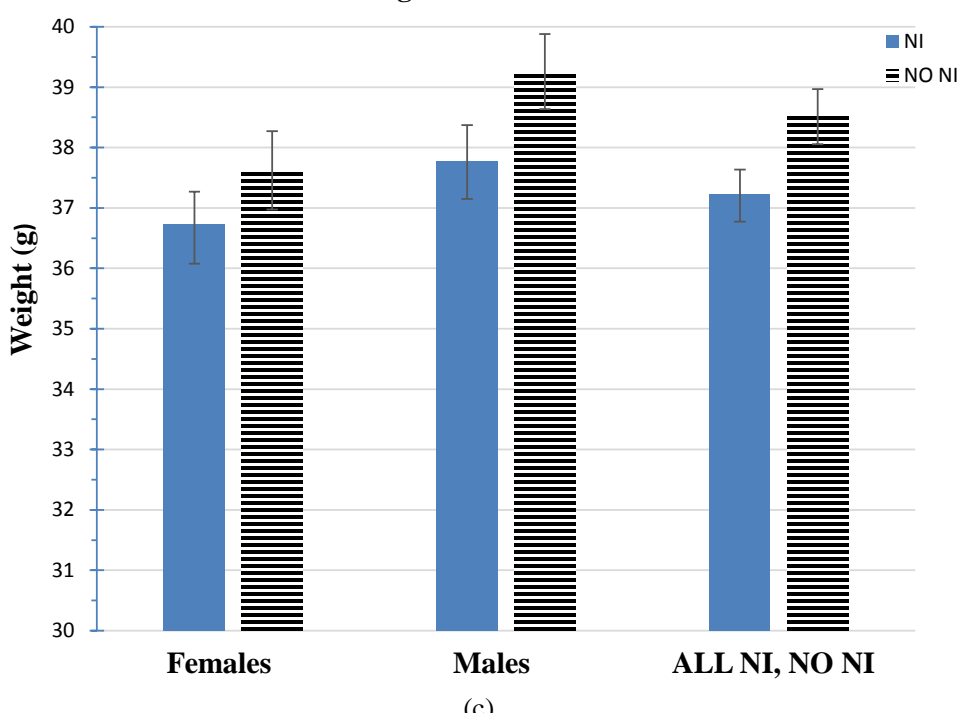

(c) 


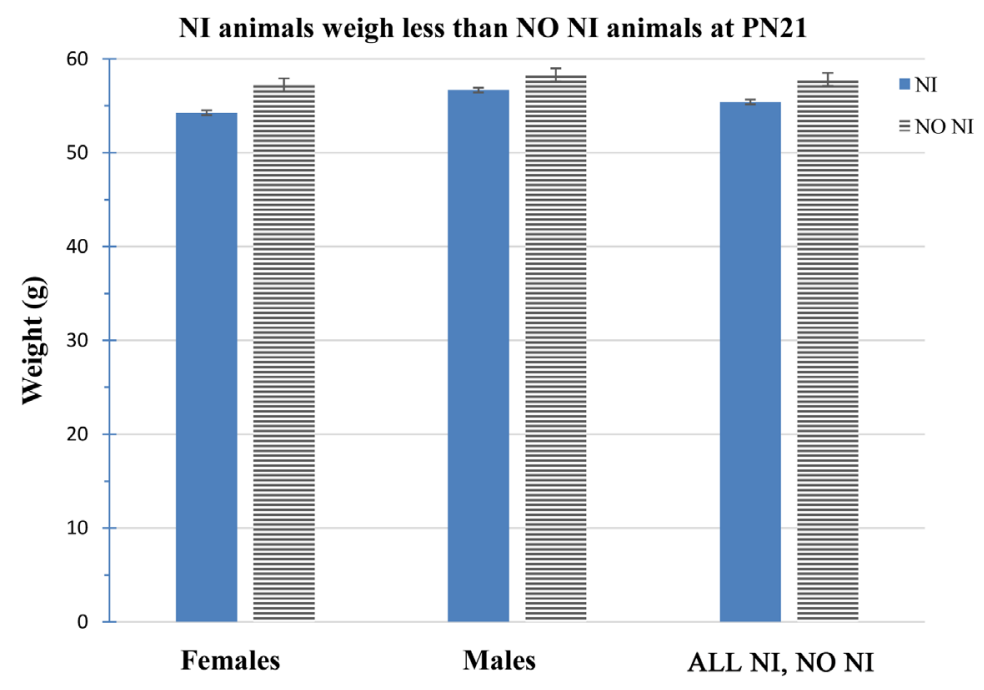

(d)

Figure 1. (a) There were no differences between NI and NO NI females or between NI and NO NI males or between environment groups overall at PN2, all $p$ 's $>0.05$. Males were heavier than females at PN2, $(\mathrm{F}(1,166)=9.983, p=$ 0.002; (b) Overall, NI animals weighed less than NO NI animals at PN7, $\mathrm{F}(1,106)=40.73, p<0.0001$, NI females weighed less than NO NI females $(\mathrm{F}(1,51)=34.818, p<0.0001)$, and NI males weighed less than NO NI males at PN7 $(F(1,55)=48.147, p=0.002$; (c) At PN14 there were no differences between NI and NO NI females or males, (all $p$ 's $>0.05)$ but overall NI animals weighed less than NO NI animals $(\mathrm{F}(1,164)=3.903, p=0.05$, Overall, males were heavier than females at PN14, $\mathrm{F}(1,164)=4.946, p=0.028$; (d) Overall, NI animals weighed less than NO NI animals at PN21, $\mathrm{F}(1,114)=7.449, p=0.007$. Overall, males were heavier than females at PN21, F $(1,114)=4.422, p=0.038$.

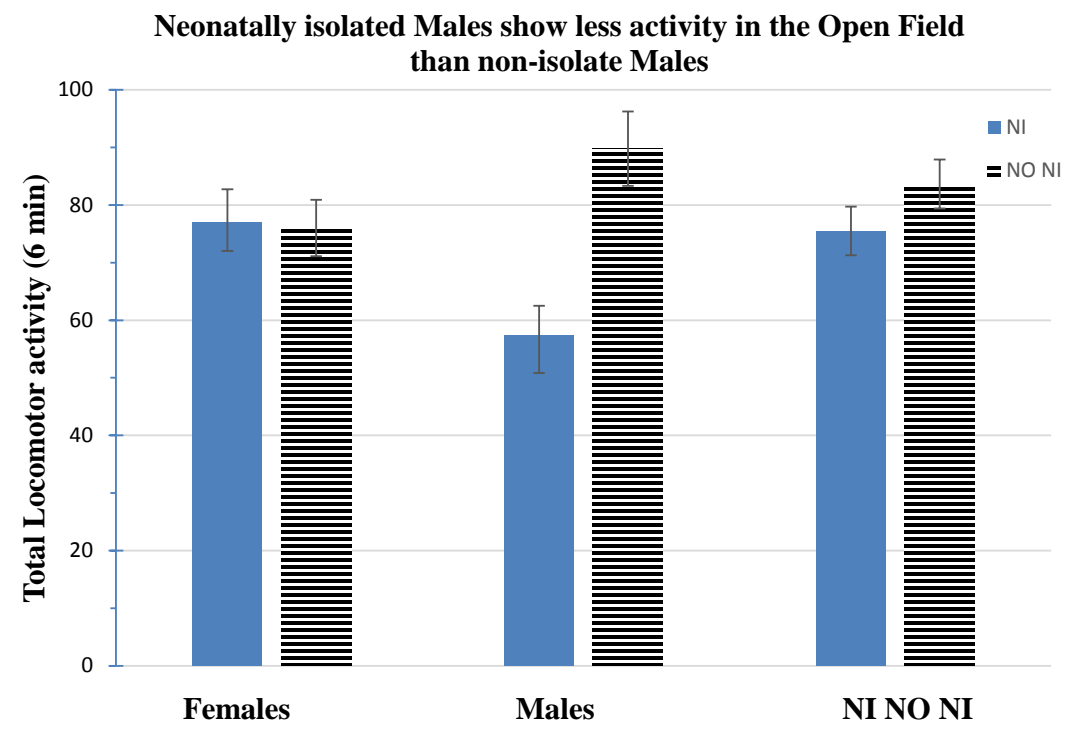

Figure 2. While there was only a trend overall for Isolates to show less horizontal activity in open field, male isolates show less activity than non-isolate males, $(\mathrm{F}(1,67)=15.516, p<0.0001)$.

mild stressor (saline injection) and immediately assessed for locomotor activity a second time in the now familiar circular corridor. Analysis revealed that overall there was a main effect of Environment, with NI animals less active following this mild stressor than NO NI animals $(F(1,44)=9.325, p=0.004$; see Figure 4). There was also a significant Sex X Environment interaction, $(\mathrm{F}(1,44)=7.236, p=0.01)$, and an additional 2 way ANOVA examining this interaction further showed that while there was no difference in activity levels of NI compared to NO NI females, NI Males were significantly less active when challenged with a mild injection stressor than the control NO NI males, $(\mathrm{F}(1,20)=13.285, p=0.002$; see Figure 4). 


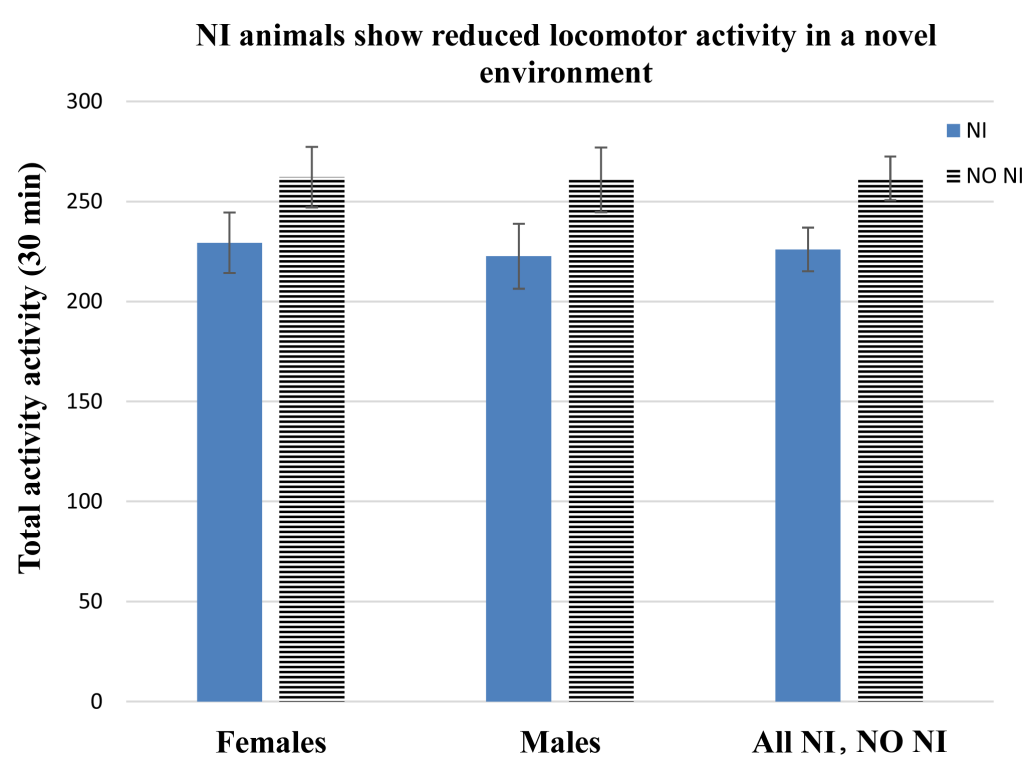

Figure 3. Overall NI rats show less total horizontal locomotor activity during a 30 minutes in a mildly stressful novel environment, the circular corridor, $(F(1,142)=6.360, p=0.013)$.

NI animals are less active after an acute mild stressor than NO NI animals

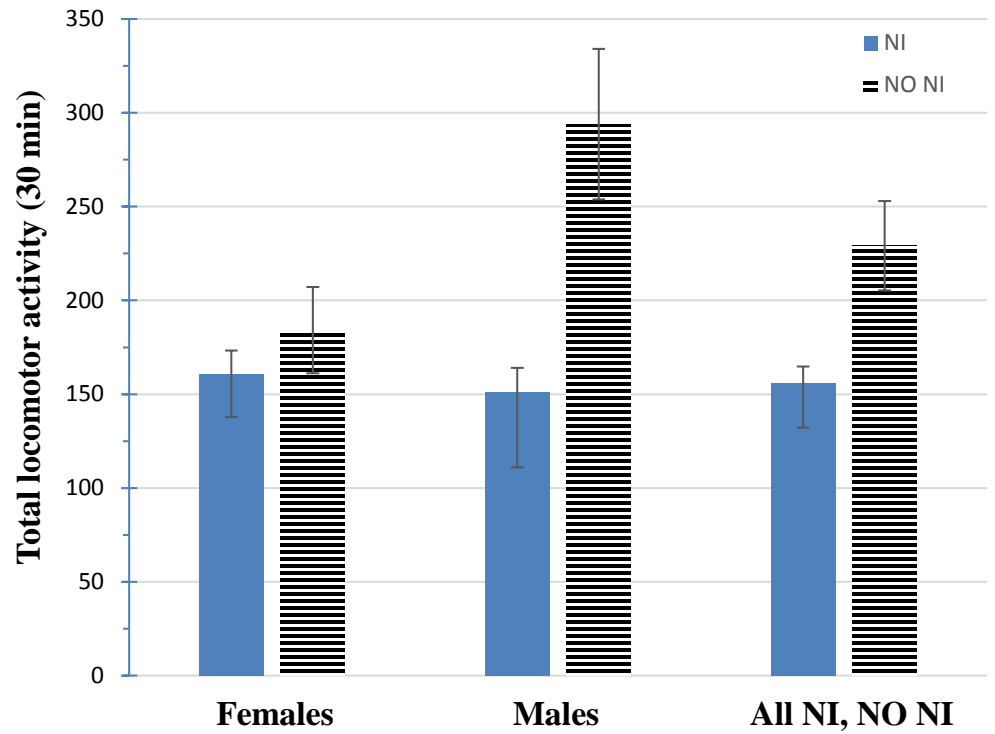

Figure 4. Overall, NI animals are less active following the mild stress of a single IP saline injection, $(\mathrm{F}(1$, $11)=9.325, p=0.004)$. NI males showed less activity following mild stress than NO NI males $(F(1,20)=$ 13.285, $p=0.002$ ); there was no effect of NI in females.

\section{Discussion}

These experiments demonstrated that exposure to the early life stress of neonatal isolation alters both physical development during the pre-weaning period and later behavioral responses to mild stress assessed during the early adolescent period.

After confirming that that no baseline Environment group differences existed prior to the Isolation manipulation, it was observed that throughout the pre-weaning period on postnatal days 7, 14, and at weaning on PN21, pups with NI experience weighed less than NO NI controls. This result is inconsistent with other reports in which neonatal isolation did not appear to affect pre-weaning weight gain. Weights assessed in isolate and 
non-isolate pre-weanling rats at PN2 and PN9 showed no group differences at those time points as a function of isolation [42]. Similarly, no effect of NI was reported in 10 day old pups [43] or at weaning on PN22 [44]. Group differences would of course not be expected at prior to the isolation manipulation beginning at PN2, but it is unclear why no isolation effects were evident at the other time points. The current results do find some support in a recent study using several different abbreviated maternal separation procedures, in which rats undergoing MS weighed less on PN5 than non-MS controls [45]. Studies have also shown that stress reduces food intake in adult male and female rats as a function of severity and duration of the stressor [46]-[48] and since NI during the SHRP is a significant stressor [49] it seems logical that stressed pre-weanling rats might gain weight more slowly than non-stressed controls. While there is no obvious resolution to this inconsistency, it should also be noted that in the present study where NI rats consistently weighed less than non-isolated counterparts, weight measures were taken on an unusually large subject sample. It may be that since the effect size (i.e., actual quantitative weight differences between groups) is quite small, as shown in Figures 1(a)-(d), only very large subject numbers would provide sufficient statistical power to reveal significant differences. Clearly additional work will need to be done to resolve these inconsistencies.

Rats tend to be neophobic, and novelty is mildly stressful [38]. Thus exposure to a novel environment activates the neuroendocrine system and is used as a non-invasive experimental manipulation to produce mild (e.g., "psychological") stress [50]-[52]. Neonatal isolation stress effects often become evident not under basal conditions but only after exposure to a stress challenge [32] so it was expected in that NI effects would be seen during exposure a novel environment. As predicted, NI exposed adolescents showed reduced locomotor activity in the novel circular corridor compared to non-isolate controls, due perhaps to an anxiety-mediated decrease of locomotor activity resulting from novelty stress in NI, but not in non-isolate animals. Although neuroendocrine changes were not assessed in the present study, it is suggested that greater HPA activation may have occurred in the NI exposed adolescent rats in response to the mild stress of the novel circular corridor. Interestingly, the observed novelty stress-induced decrement in activity was carried by males, since NI males, but not NI females, showed a significant decrease in locomotor activity in the novel environment compared to their non-isolate controls. In prior work, adult male and female rats with neonatal isolation experience tested in a novel locomotor apparatus showed no overall effect of NI, and no difference in activity level between males and females [18]. These reported differences in stress-mediated activity changes in NI exposed adult and adolescent rats in response to a novel environment might be due at least in part to inherent differences in adults vs. adolescents in emotional reactivity to mild stress, activity level, or basal anxiety levels. Studies comparing rats of different ages report that behaviors such as locomotor and exploratory activity, as well as reward processing and emotional responding, change non-linearly as a function of age. Specifically, when adolescent rats were compared to younger animals and to adults, changes in these characteristics followed an inverted U-shape pattern, with the peak occurring during adolescence [53]. Perhaps the greater inherent predisposition of adolescents toward higher levels of exploratory and locomotor activity renders these measures more sensitive to experimental perturbation in adolescents. Alternatively, greater emotional reactivity in adolescents compared to adults might explain the greater efficacy of NI in causing stress-induced suppression of activity in adolescents, while having little or no effect in adults.

McCormick et al. (2002; [32]) examined NI effects on locomotor activity in male and female juveniles following either 1 hour restraint stress or no restraint; the non-restrained animals were thus directly comparable in early life stress treatment, age, and sex to subjects in the current study. As in the present study, animals showed no sex differences in locomotor activity, but unlike the present study, also showed no NI-induced decrease in locomotor activity. This is very likely due to a small but important procedural difference between the two studies. McCormick et al. (2002; [32]) exposed subjects to the locomotor apparatus one day prior to the actual locomotor activity measurement to habituate them to the apparatus. By contrast, locomotor activity in the current study was assessed during initial exposure to the apparatus, so the environment was novel at testing. In eliminating the novelty factor and its associated stress by habituation, McCormick et al. (2002; [32]) may also have eliminated an anxiety-mediated reduction of activity in the NI animals.

Like initial exposure to a novel environment, e.g., the novel circular corridor, intraperitoneal (IP) saline injection is a mild stressor [41] and was expected to induce greater stress and therefore affect locomotor activity differently in animals with neonatal isolation experience. A subset of the animals tested previously in the circular corridor were randomly chosen and assessed for locomotor activity a second time in the now familiar circular corridor immediately after receiving an acute IP saline injection. As expected, effects of the injection were con- 
sistent with results seen during initial exposure to the novel circular locomotor environment; overall, males and females displayed similar levels of activity and again, locomotor activity was reduced in isolate animals compared to non-isolates. This effect was again carried by males, with NI exposed males showing far less locomotor activity after IP injection than non-isolate males, and no differences in activity seen in females. The observation here that NI appears to have greater impact on stress-mediated behaviors in males than females is consistent with other studies from our lab (unpublished data) that found sexually dimorphic effects of NI in early adolescent male and female rats on several additional stress sensitive behavioral measures.

Other studies utilizing IP saline injection as a mild stressor vary greatly in methodologies from the single challenge injection used here, and from one another, as do the reported effects. For example, when a compound stimulus comprised of IP saline injection plus crowding was administered repeatedly to pregnant dams, adult male progeny showed increased locomotor activity in an open field, presumably reflecting decreased stress [54]. Conversely, IP saline alone administered repeatedly and prenatally was observed to produce adult male progeny that displayed more floating behavior and more time struggling during a swim test, and decreased rearing and locomotor activity in the open field, all indicative of increased stress [55]. In work somewhat more comparable methodologically to the present study, 24 hour maternal deprivation during the SHRP (PN11) resulted in juvenile rats showing more center locomotor activity and reduced IP saline injection-induced corticosterone compared with non-maternally deprived controls [56]. These results suggest that this early life stressor reduced stress in the open field and in response to IP saline injection, contrary to what was observed in the present study. This may be yet one more example of different early life stress procedures resulting in diametrically different neuroendocrine and behavioral outcomes. Maternal separation methodologies vary widely, for example, in age of exposure, duration and intensity, and so produce inconsistent results compared to both other studies using maternal separation and those utilizing the neonatal isolation procedure. It is not surprising, therefore, that 24 hours maternal deprivation resulted in juvenile rats expressing reduced anxiety, while neonatal isolation resulted in indications of increased stress in early adolescent rats herein.

\section{Conclusion}

In conclusion, an extensive literature encompassing many varied early life stress procedures suggests that adverse experience during the pre-weaning period, and especially during the SHRP affects the development and function of neurochemical, neurophysiological and neuroendocrine systems. Most of the literature involves studies with adult rats, so is also well known that the impact of those early life stressors endures and manifests later in life in the adult. Far less work has been done on other aged groups. Adolescent rats differ greatly from adult animals in both brain and behavior [53] [57], and in order to more fully understand the effects of early life stress on adolescent aged animals, additional work with this age group is needed. The present study contributes evidence to a smaller but growing literature examining neonatal isolation stress effects in early adolescence, and illustrates some of the similarities and differences compared with NI impact in adult animals. Future work will continue to examine early life stress manipulations in adolescence, with particular focus on sexually dimorphic effects of neonatal isolation as they were suggested here.

\section{References}

[1] Kehoe, P. and Shoemaker, W.J. (2001) Infant Stress, Neuroplasticity, and Behavior. In: Blass, E., Ed., Developmental Psychobiology, Vol. 13, Kluwer Academic/Plenum, New York, 551-585. http://dx.doi.org/10.1007/978-1-4615-1209-7_15

[2] Ladd, C.O., Huot, R.L., Thrivikraman, K.V., Nemeroff, C.B., Meaney, M.J. and Plotsky, P.M. (2000) Long-Term Behavioral and Neuroendocrine Adaptations to Adverse Early Experience. Progress in Brain Research, 122, 81-103. http://dx.doi.org/10.1016/S0079-6123(08)62132-9

[3] Anisman, H., Zaharia, M.D., Meaney, M.J. and Merali, Z. (1998) Do Early Life Events Permanently Alter Behavioral and Hormonal Responses to Stressors? International Journal of Developmental Neuroscience, 16, 149-164. http://dx.doi.org/10.1016/S0736-5748(98)00025-2

[4] Macri, S. and Würbel, H. (2006) Developmental Plasticity of HPA and Fear Responses in Rats: A Critical Review of the Maternal Mediation hypothesis. Hormones and Behavior, 50, 667-680. http://dx.doi.org/10.1016/j.yhbeh.2006.06.015

[5] Sapolsky, R.M. and Meaney, M.J. (1986) Maturation of the Adrenocortical Stress Response: Neuroendocrine Control Mechanisms and the Stress Hyporesponsive Period. Brain Research Reviews, 11, 65-76. 
http://dx.doi.org/10.1016/0165-0173(86)90010-X

[6] Pryce, C.R. and Feldon, J. (2003) Long-Term Neurobehavioural Impact of the Postnatal Environment in Rats: Manipulations, Effects and Mediating Mechanisms. Neuroscience \& Biobehavioral Reviews, 27, 57-71. http://dx.doi.org/10.1016/S0149-7634(03)00009-5

[7] Levine, S. (2001) Primary Social Relationships Influence the Development of the Hypothalamic-Pituitary-Adrenal Axis in the Rat. Physiology \& Behavior, 73, 255-260. http://dx.doi.org/10.1016/S0031-9384(01)00496-6

[8] Cirulli, F., Gottlieb, S.L., Rosenfeld, P. and Levine, S. (1992) Maternal Factors Regulate Stress Responsiveness in the Neonatal Rat. Psychobiology, 20, 143-152.

[9] Knuth, E.D. and Etgen, A.M. (2007) Long-Term Behavioral Consequences of Brief, Repeated Neonatal Isolation. Brain Research, 1128, 139-147. http://dx.doi.org/10.1016/j.brainres.2006.10.054

[10] Kalinichev, M., Easterling, K.W., Plotsky, P.M. and Holtzman, S.G. (2002) Long-Lasting Changes in Stress-Induced Corticosterone Response and Anxiety-Like Behaviors as a Consequence of Neonatal Maternal Separation in LongEvans Rats. Pharmacology Biochemistry and Behavior, 73, 131-140. http://dx.doi.org/10.1016/S0091-3057(02)00781-5

[11] Wigger, A. and Neumann, I.D. (1998) Periodic Maternal Deprivation Induces Gender-Dependent Alterations in Behavioral and Neuroendocrine Responses to Emotional Stress in Adults. Physiology and Behavior, 66, 293-302. http://dx.doi.org/10.1016/S0031-9384(98)00300-X

[12] Imanaka, A., Morinobu, S., Toki, S. and Yamawaki, S. (2006) Importance of Early Environment in the Development of Post-Traumatic Stress Disorder-Like Behaviors. Behavioral Brain Research, 173, 129-137. http://dx.doi.org/10.1016/j.bbr.2006.06.012

[13] Meaney, M.J., Mitchell, J.B., Aitken, D.H., Bhatnagar, S., Bodnoff, S.R., Iny, L.J. and Sarrieau, A. (1991) The Effects of Neonatal Handling on the Development of the Adrenocortical Response to Stress: Implications for Neuropathology and Cognitive Deficits in Later Life. Psychoneuroendocrinology, 16, 85-103. http://dx.doi.org/10.1016/0306-4530(91)90072-2

[14] Lai, M.-C. and Huang, L.-T. (2011) Effects of Early Life Stress on Neuroendocrine and Neurobehavior: Mechanisms and Implications. Pediatrics and Neonatology, 52, 122-129. http://dx.doi.org/10.1016/j.pedneo.2011.03.008

[15] Kehoe, P., Shoemaker, W.J., Triano, L., Callahan, M. and Rappolt, G. (1998) Adult Rats Stressed as Neonates Show Exaggerated Behavioral Responses to Both Pharmacological and Environmental Challenges. Behavioral Neuroscience, 112, 116-125. http://dx.doi.org/10.1037/0735-7044.112.1.116

[16] Brake, W.G., Zhang, T.Y., Diorio, J., Meaney, M.J. and Gratton, A. (2004) Influence of Early Postnatal Rearing Conditions on Mesocorticolimbic Dopamine and Behavioral Responses to Psychostimulants and Stressors in Adult Rats. European Journal of Neuroscience, 19, 1863-1874. http://dx.doi.org/10.1111/j.1460-9568.2004.03286.x

[17] Lynch, W.J., Mangini, L.D. and Taylor, J.R. (2005) Neonatal Isolation Stress Potentiates Cocaine Seeking Behavior in Adult Male and Female Rats. Neuropsychopharmacology, 30, 322-329. http://dx.doi.org/10.1038/sj.npp.1300594

[18] Kosten, T.A., Miserendino, M.J.D. and Kehoe, P. (2000) Enhanced Acquisition of Cocaine Self-Administration in Adult Rats with Neonatal Isolation Stress Experience. Brain Research, 875, 44-50.

[19] Kosten, T.A., Sanchez, H., Zhang, X.Y. and Kehoe, P. (2004) Neonatal Isolation Enhances Acquisition of Cocaine Self-Administration and Food Responding in Female Rats. Behavioural Brain Research, 151, 137-149.

[20] Zhang, X.Y., Sanchez, H., Kehoe, P. and Kosten, T.A. (2005) Neonatal Isolation Enhances Maintenance but Not Reinstatement of Cocaine Self-Administration in Adult Male Rats. Psychopharmacology, 177, 391-399. http://dx.doi.org/10.1007/s00213-004-1963-y

[21] Kosten, T.A., Zhang, X.Y. and Kehoe, P. (2005) Heightened Cocaine and Food Self-Administration in Female Rats with Neonatal Isolation Experience. Neuropsychopharmacology, 31, 70-76. http://dx.doi.org/10.1038/sj.npp.1300779

[22] Kosten, T.A., Zhang, X.-Y. and Kehoe, P. (2005) Neurochemical and Behavioral Responses to Cocaine in Adult Male Rats with Neonatal Isolation Experience. Journal of Pharmacology and Experimental Therapeutics, 314, 661-667. http://dx.doi.org/10.1124/jpet.104.081216

[23] Kosten, T.A., Kim, J.J. and Lee, H.J. (2012) Early Life Manipulations Alter Learning and Memory in Rats. Neuroscience \& Biobehavioral Reviews, 36, 1985-2006. http://dx.doi.org/10.1016/j.neubiorev.2012.07.003

[24] Zhang, X., Wang, B., Jin, J., An, S., Zeng, Q., Duan, Y., et al. (2014) Early Deprivation Reduced Anxiety and Enhanced Memory in Adult Male Rats. Brain Research Bulletin, 108, 44-50. http://dx.doi.org/10.1016/j.brainresbull.2014.08.005

[25] Brunson, K.L., Chen, Y., Avishai-Einer, S. and Baram, T.Z. (2003) Stress and the Developing Hippocampus. Molecular Neurobiology, 27, 121-136. http://dx.doi.org/10.1385/MN:27:2:121

[26] Kosten, T.A., Karanian, D.A., Yeh, J., Haile, C.A., Kim, J.J., Kehoe, P. and Bahr, B.A. (2007) Memory Impairments 
and Hippocampal Modifications in Adult Rats with Neonatal Isolation Stress Experience. Neurobiology of Learning and Memory, 88, 167-176. http://dx.doi.org/10.1016/j.nlm.2007.03.011

[27] Lehmann, J. and Feldon, J. (2000) Long-Term Bio-Behavioural Effects of Maternal Separation in the Rat: Consistent or Confusing? Reviews in the Neurosciences, 11, 383-408. http://dx.doi.org/10.1515/REVNEURO.2000.11.4.383

[28] Kehoe, P., Hoffman, J.H., Austin-Lafrance, R.J. and Bronzino, J.D. (1995) Neonatal Isolation Enhances Hippocampal Dentate Response to Tetanization in Freely Moving Juvenile Male Rats. Experimental Neurology, 136, 89-97. http://dx.doi.org/10.1006/exnr.1995.1086

[29] McCormick, C.M. (2010) An Animal Model of Social Instability Stress in Adolescence and Risk for Drugs of Abuse. Physiology \& Behavior, 99, 194-203.

[30] Anderson, S.L. (2003) Trajectories of Brain Development: Point of Vulnerability or Window of Opportunity? Neuroscience \&Biobehavioral Reviews, 27, 3-18. http://dx.doi.org/10.1016/S0149-7634(03)00005-8

[31] Kehoe, P., Shoemaker, W.J., Triano, L., Hoffman, J. and Arons, C. (1996) Repeated Isolation in the Neonatal Rat Produces Alterations in Behavior and Ventral Striatal Dopamine Release in the Juvenile after Amphetamine Challenge. Behavioral Neuroscience, 110, 1435-1444. http://dx.doi.org/10.1037/0735-7044.110.6.1435

[32] McCormick, C.M., Kehoe, P., Mallinson, K., Cecchi, L. and Frye, C.A. (2002) Neonatal Isolation Alters Stress Hormone and Mesolimbic Dopamine Release in Juvenile Rats. Pharmacology Biochemistry and Behavior, 73, 77-85.

[33] Bronzino, J.D., Kehoe, P., Austin-LaFrance, R.J., Rushmore, R.J. and Kurdian, J. (1996) Neonatal Isolation Alters LTP in Freely Moving Juvenile Rats: Sex Differences. Brain Research Bulletin, 41, 175-183. http://dx.doi.org/10.1016/0361-9230(96)00166-9

[34] Kosten, T.A., Zhang, X.Y. and Kehoe, P. (2003) Chronic Neonatal Isolation Stress Enhances Cocaine-Induced Increases in Ventral Striatal Dopamine Levels in Rat Pups. Developmental Brain Research, 141, 109-116. http://dx.doi.org/10.1016/S0165-3806(03)00003-8

[35] Doremus-Fitzwater, T.L., Varlinskaya, E.I. and Spear, L.P. (2009) Effects of Pretest Manipulation on Elevated Plus-Maze Behavior in Adolescent and Adult Male and Female Sprague-Dawley Rats. Pharmacology Biochemistry and Behavior, 92, 413-423.

[36] Doyle, G. and Yule, E.P. (1959) Grooming Activities and Freezing Behavior in Relation to Emotionality in Albino Rats. Animal Behaviour, 7, 18-22. http://dx.doi.org/10.1016/0003-3472(59)90025-9

[37] Mead, A.P. (1960) A Quantitative Method for the Analysis of Exploratory Behavior in the Rat. Animal Behaviour, 8, 19-31. http://dx.doi.org/10.1016/0003-3472(60)90005-1

[38] Ennaceur, A., Michalikova, S. and Chazot, P.L. (2006) Models of Anxiety: Responses of Rats to Novelty in an Open Space and an Enclosed Space. Behavioural Brain Research, 171, 26-49. http://dx.doi.org/10.1016/j.bbr.2006.03.016

[39] Treit, D. and Fundytus, M. (1988) Thigmotaxis as a Test for Anxiolytic Activity in Rats. Pharmacology Biochemistry and Behavior, 31, 959-962. http://dx.doi.org/10.1016/0091-3057(88)90413-3

[40] Piazza, P.V., Deminiere, J.M., Le Moal, M. and Simon, H. (1989) Factors That Predict Individual Vulnerability to Amphetamine Self-Administration. Science, 245, 1511-1513. http://dx.doi.org/10.1126/science.2781295

[41] Dilsaver, S.C. and Majchrzak, M.J. (1990) Effects of Placebo (Saline) Injections on Core Temperature in the Rat. Progress in Neuro-Psychopharmacology and Biological Psychiatry, 14, 417-422. http://dx.doi.org/10.1016/0278-5846(90)90029-G

[42] Lai, M.-C., Yang, S.-N. and Huang, L.-T.(2008) Neonatal Isolation Enhances Anxiety-Like Behavior Following Early-Life Seizure in Rats. Pediatrics \& Neonatology, 49, 19-25. http://dx.doi.org/10.1016/S1875-9572(08)60006-6

[43] Huang, L.T., Holmes, G.L., Lai, M.C., Hung, P.L., Wang, C.L., Wang, T.J., Yang, C.H., Liou, C.W. and Yang, S.N. (2002) Maternal Deprivation Stress Exacerbates Cognitive Deficits in Immature Rats with Recurrent Seizures. Epilepsia, 43, 1141-1148. http://dx.doi.org/10.1046/j.1528-1157.2002.14602.x

[44] Imanaka, A., Morinobu, S., Toki, S., Yamamoto, S., Matsuki, A., Kozuru, T. and Yamawaki, S. (2008) Neonatal Tactile Stimulation Reverses the Effect of Neonatal Isolation on Open-Field and Anxiety-Like Behavior, and Pain Sensitivity in Male and Female Adult Sprague-Dawley Rats. Behavioural Brain Research, 186, 91-97. http://dx.doi.org/10.1016/j.bbr.2007.07.039

[45] Daskalakis, N.P., Claessens, S.E.F., Laboyrie, J.L.L., Enthoven, L., Oitzl, M.S., Champagne, D.L. and de Kloet, E.R. (2011) The Newborn Rat's Stress System Readily Habituates to Repeated and Prolonged Maternal Separation, While Continuing to Respond to Stressors in Context Dependent Fashion. Hormones and Behavior, 60, 165-176. http://dx.doi.org/10.1016/j.bbr.2007.07.039

[46] Marti, O., Marti, J. and Armario, A. (1994) Effects of Chronic Stress on Food Intake in Rats: Influence of Stressor Intensity and Duration of Daily Exposure. Physiology \& Behavior, 55, 747-753. 
http://dx.doi.org/10.1016/0031-9384(94)90055-8

[47] Vallès, A., Martí, O., García, A. and Armario, A. (2000) Single Exposure to Stressors Causes Long-Lasting, StressDependent Reduction of Food Intake in Rats. American Journal of Physiology—Regulatory Integrative and Comparative Physiology, 279, 1138-1144.

[48] Bali, A. and Jaggi, A.S. (2015) Preclinical Experimental Stress Studies: Protocols, Assessment and Comparison. European Journal of Pharmacology, 746, 282-292. http://dx.doi.org/10.1016/j.ejphar.2014.10.017

[49] Antoniazzia, C.T.D., Boufleura, N., Pasea, C.S., Kuhna, F.T., Diasb, V.T., Segatb, H.J., et al. (2014) Tactile Stimulation and Neonatal Isolation Affect Behavior and Oxidative Status Linked to Cocaine Administration in Young Rats. Behavioural Processes, 103, 297-305. http://dx.doi.org/10.1016/j.beproc.2014.01.011

[50] Pfister, H.P. (1979) The Glucocorticosterone Response to Novelty as a Psychological Stressor. Physiology \& Behavior, 23, 649-652. http://dx.doi.org/10.1016/0031-9384(79)90154-9

[51] Rothwell, P.E., Kourrich, S. and Thomas, M.J. (2001) Environmental Novelty Causes Stress-Like Adaptations at Nucleus Accumbens Synapses: Implications for Studying Addiction-Related Plasticity. Neuropharmacology, 61, 11521159.

[52] Márquez, C., Nadal, R. and Armario, A. (2005) Responsiveness of the Hypothalamic-Pituitary-Adrenal Axis to Different Novel Environments Is a Consistent Individual Trait in Adult Male Outbred Rats. Psychoneuroendocrinology, 30, 179-187. http://dx.doi.org/10.1016/j.psyneuen.2004.05.012

[53] Brenhouse, H.C. and Andersen, S.L. (2011) Developmental Trajectories during Adolescence in Males and Females: Across-Species Understanding of Underlying Brain Changes. Neuroscience \& Biobehavioral Reviews, 35, 1687-1703. http://dx.doi.org/10.1016/j.neubiorev.2011.04.013

[54] Peters, D.A. (1986) Prenatal Stress Increases the Behavioral Response to Serotonin Agonists and Alters Open Field Behavior in the Rat. Pharmacology Biochemistry and Behavior, 25, 873-877. http://dx.doi.org/10.1016/0091-3057(86)90400-4

[55] Šlamberová, R., Schindler, C.J. and Vathya, I. (2002) Impact of Maternal Morphine and Saline Injections on Behavioral Responses to a Cold Water Stressor in Adult Male and Female Progeny. Physiology \& Behavior, 75, 723-732. http://dx.doi.org/10.1016/S0031-9384(02)00669-8

[56] Suchecki, D., Palma, B.D. and Tufik, S. (2000) Pituitary-Adrenal Axis and Behavioural Responses of Maternally Deprived Juvenile rats to the Open Field. Behavioural Brain Research, 111, 199-106. http://dx.doi.org/10.1016/s0166-4328(00)00148-0

[57] Spear, L.P. (2000) The Adolescent Brain and Age-Related Behavioral Manifestations. Neuroscience and Biobehavioral Reviews, 24, 417-463. http://dx.doi.org/10.1016/S0149-7634(00)00014-2 